\title{
Effects of experimental hyperlipoproteinaemia on the retina and optic nerve in rhesus monkeys
}

\author{
LUTZA YANKO,${ }^{14}$ ISAAC C. MICHAELSON,${ }^{4}$ ELIEZER ROSENMANN,${ }^{2}$ \\ MOSHE IVRI, ${ }^{1}$ AND IRVING LUTSKY ${ }^{3}$ \\ From the Departments of ${ }^{1}$ Ophthalmology, ${ }^{2}$ Pathology, and ${ }^{3}$ Comparative Medicine, \\ Hadassah University Hospital, and the ${ }^{4}$ Hebrew University-Hadassah Medical School and the \\ Jerusalem Institute for the Prevention of Blindness, Jerusalem, Israel
}

SUMMARY Hyperlipoproteinaemia resulting from thyroid suppression and long-term ingestion of a high cholesterol diet caused prolonged lipaemia retinalis in 6 rhesus monkeys. No atherosclerotic deposits or other ophthalmoscopically visible changes of the retinal vasculature were detectable. In 2 animals histopathological examination revealed bilateral segmental atrophy and gliosis of the optic nerves bearing a resemblance to chronic ischaemic optic neuropathy. One monkey developed ophthalmoscopically visible temporal pallor of the optic discs. Emboli of fat laden cells in blood vessels of the brain in one of these 2 monkeys, and in a penicillated splenic artery in the other animal, were associated with signs of systemic embolic occlusion, suggesting a similar course in the development of optic nerve damage.

The systemic effects of diet-induced hyperlipoproteinaemia have been extensively studied in rhesus monkeys ${ }^{1-3}$ as well as in other primate species. ${ }^{4}$ The ocular effects of experimentally induced hypercholesterolaemia have been assessed mainly in rabbits ${ }^{56}$ and dogs $^{7}$ and have usually involved the anterior segment of the eye.

In an effort to produce alterations in the ocular vasculature in primates we subjected hypothyroid monkeys to a dietary regimen consisting of high fat and high cholesterol for up to 16 months. In this report we describe the effects of this diet on the optic nerve and retina.

\section{Materials and methods}

Animals and housing. The experimental animals included 6 healthy female rhesus monkeys (Macaca mulatta) obtained as adults from the Israel Biological Institute, Ness Ziona. Age was estimated by body weight (3.7-8.0 kg) and eruption of the third molar teeth. A pre-experimental conditioning period of 6 to 8 weeks included testing for tuberculosis and deworming. All animals were individually housed in suspended cages in an animal room maintained at $24^{\circ}-27^{\circ} \mathrm{C}$ and lighted from 6.00 a.m. to 6.00 p.m.

Correspondence to Lutza Yanko, MD, Department of Ophthalmology, Hadassah University Hospital, Jerusalem, Israel.
Water was available continuously, and food was replenished twice daily.

Thyroid function suppression. After the conditioning period each monkey was rendered hypothyroid by the intraperitoneal injection of radioactive iodine $\left({ }^{131} \mathrm{I}\right), 1 \mathrm{mCi} / \mathrm{kg}$ body weight) in an attempt to achieve higher levels of hypercholesterolaemia. ${ }^{3}$ Chemical suppression of the thyroid was followed by a period of at least 4 weeks prior to institution of the high lipid diet.

Diet. Cholesterol powder and melted lard were mixed with pellets of a complete laboratory animal chow (Amrod 931, Ambar Feed Mills, Hadera, Israel) so that the resulting diet contained $10 \%$ cholesterol and $20 \%$ total fat on analysis. A 30-day supply of food was thus prepared, stored under refrigeration $\left(4^{\circ} \mathrm{C}\right)$, and analysed routinely for total fat cholesterol content. The amount of food consumed daily was estimated and recorded.

Physical and biochemical determinations. Under general anaesthesia (ketamine $\mathrm{HC} 115 \mathrm{mg} / \mathrm{kg}$ body weight intramuscularly) ocular examinations and fundus photography were carried out. At the same time as blood samples were drawn for serum cholesterol, triglycerides, and selected biochemical studies, all animals were weighed. Reproducible measurements of indirect blood pressure were not technically feasible in this study. 
Table 1 Summary of experimental data of 6 hypothyroid female monkeys receiving a high cholesterol and high fat diet

\begin{tabular}{|c|c|c|c|c|c|c|c|c|}
\hline \multirow{2}{*}{$\begin{array}{l}\text { Number } \\
\text { of } \\
\text { animal }\end{array}$} & \multirow{2}{*}{$\begin{array}{l}\text { Period on } \\
\text { diet } \\
\text { (months) }\end{array}$} & \multicolumn{2}{|c|}{ Weight, $k g$} & \multicolumn{3}{|c|}{ Serum cholesterol } & \multicolumn{2}{|c|}{ Serum triglycerides } \\
\hline & & Initial & Terminal & $\begin{array}{l}\text { Before } \\
\text { diet }\end{array}$ & $\begin{array}{l}\text { Average } \\
\text { during } \\
\text { diet }\end{array}$ & $\begin{array}{l}\text { Highest level } \\
\text { during diet }\end{array}$ & $\begin{array}{l}\text { Average } \\
\text { during } \\
\text { diet }\end{array}$ & $\begin{array}{l}\text { Highest level } \\
\text { during diet }\end{array}$ \\
\hline $1 \mathrm{C}$ & 16 & $8 \cdot 0$ & $5 \cdot 8$ & 165 & 1540 & 2297 & 1406 & 4000 \\
\hline $2 \mathrm{C}$ & 9 & 3.9 & 3.6 & 150 & 1463 & 2736 & 1849 & 3192 \\
\hline $12 \mathrm{C}$ & 13 & 3.7 & 3.4 & 150 & 1872 & 3048 & 1864 & 2653 \\
\hline $43 C$ & 14 & $4 \cdot 0$ & $3 \cdot 8$ & 240 & 1454 & 3485 & 1290 & 2812 \\
\hline $30 \mathrm{D}$ & 13 & 6.4 & $4 \cdot 3$ & 130 & 1390 & 2812 & 2030 & 3049 \\
\hline $14 E$ & 14 & $4 \cdot 2$ & $3 \cdot 2$ & 135 & 2228 & 3554 & 2848 & 5782 \\
\hline
\end{tabular}

SI conversion: serum cholesterol $\mathrm{mg} / \mathrm{dl} \times 0.026=\mathrm{mmol} / \mathrm{l}$; serum triglycerides $\mathrm{mg} / \mathrm{dl} \times 0.0113=\mathrm{mmol} / \mathrm{l}$.

Post-mortem evaluation. Complete necropsies were performed on 3 of 5 monkeys which died during the study, and on one animal that was killed. Tissue samples of all organs were fixed in $10 \%$ formalin, embedded in paraffin, and sections $(6 \mu \mathrm{m})$ stained with haematoxylin and eosin. Selected frozen sections were stained with oil red-O for neutral fats.

The eyes were removed with 6 to $8 \mathrm{~mm}$ of optic nerve attached and immersed in $10 \%$ formalin. After fixation the globes were divided in the horizontal plane and the retinae were examined with a stereo microscope. Serial paraffin sections were stained with haematoxylin and eosin, periodic acid-Schiff (PAS) reaction, Mallory's phosphotungstic acid haematoxylin method for connective tissues, Bodian method for axis cylinders, Luxol fast blue-PAS for myelin, Luxol fast blue, and alcian blue for acid mucopolysaccharides. Frozen sections were stained with oil-red $O$.

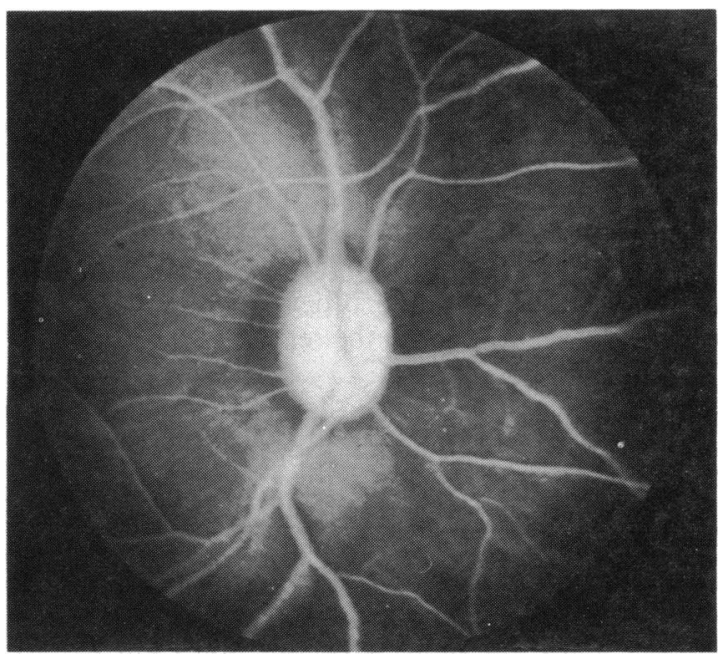

Fig. 1 Lipaemia retinalis in the right eye of a monkey (12C) fed a high fat-cholesterol diet for 13 months.

\section{Results}

GENERAL OBSERVATIONS

Body weights of the monkeys at the outset and at the end of the study, the number of months on high cholesterol diet, and serum cholesterol and triglycerides levels are presented in Table 1.

All animals lost weight during the experimental period. One monkey (2C) developed a diffuse central neuron motor paresis and died suddenly 9 months after starting the high lipid diet. Another monkey (12C) died from sepsis 13 months after starting the experiment. Two animals (30D and 43C) were examined post mortem at 13 and 14 months. Severe post-mortem autolysis in 2 monkeys found dead in their cages precluded post-mortem examination.

Serum lipids. During the study mean serum cholesterol levels ranged between 1390 and $2228 \mathrm{mg} / \mathrm{dl}$ as compared with pretreatment levels of 130 to 240 $\mathrm{mg} / \mathrm{dl}$. Peak levels of serum cholesterol from 2297 to $3554 \mathrm{mg} / \mathrm{dl}$ were recorded. Mean values for serum triglycerides in the animals studied ranged from 1290 to $2848 \mathrm{mg} / \mathrm{dl}$, and peak triglyceride levels between 2653 and $5782 \mathrm{mg} / \mathrm{dl}$ were observed (Table 1 ). (SI conversion: $\mathrm{mg} / \mathrm{dl} \times 0 \cdot 026=\mathrm{mmol} / \mathrm{l}$.)

\section{OPHTHALMOSCOPIC FINDINGS}

Direct and indirect ophthalmoscopic examinations and colour diapositives of the fundi revealed the presence of lipaemia retinalis in all the animals. There was a marked change in the colour of the retinal vessels, which appeared as if they contained a milky white or yellow-hued substance (Fig. 1). Occasionally both the arteries and veins were uniform in appearance and difficult to distinguish by colour alone. These ophthalmoscopic features were strikingly apparent in the early stages of hyperlipoproteinaemia in the peripheral retinal vessels. However, as the cholesterol and triglyceride levels increased, the yellowish colour of the vessels in the posterior pole approached that seen at the periphery. Another prominent ophthal- 
Fig. 2 Prelaminar and retrolaminar atrophy of temporal nerve tissue and gliosis of the optic nerve head of a monkey fed a high fat-cholesterol diet for 9 months. (Phosphotungstic acid haematoxylin, $\times 53$ ).

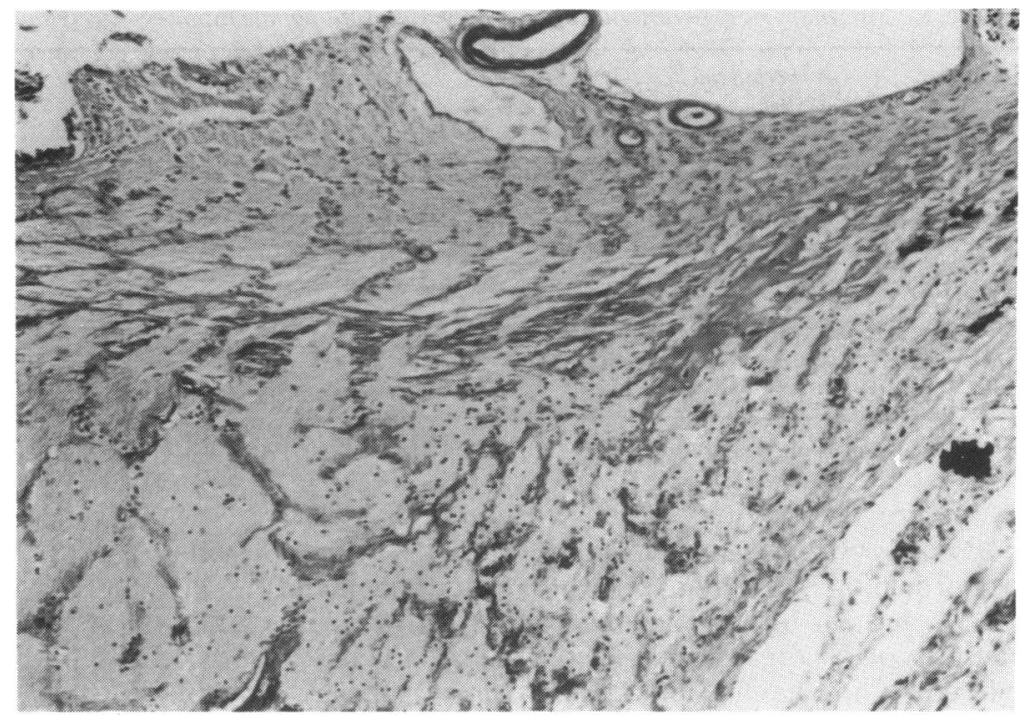

moscopic change evident throughout the study was temporal pallor of the optic discs in one monkey (2C).

Small discrete spheroid bodies, namely, asteroids, creamy and shiny in appearance, floating in the vitreous in all directions, were present in both eyes of monkey 1C. These vitreous features bear a strong resemblance to clinical asteroid hyalitis.

\section{POST-MORTEM FINDINGS}

Systemic pathological findings. Prominent pathological findings in the 4 monkeys examined postmortem were severe diffuse fatty changes of the liver. Xanthomas were found on the skin in 2 monkeys (12C, 30D), in which xanthomatous transformation of endothelial cells of splenic sinusoids was seen in one case, and renal corpuscles with xanthomatous transformation of glomerular endothelial cells in the other. In 2 instances embolisation by fat laden cells was noted: in one animal (2C) in the vessels of the brain affecting numerous areas, with ensuing multifocal haemorrhagic necrosis; and in the other animal (43C) in a penicillated splenic artery, leading to occlusive thrombus formation.

In one monkey (12C) septic pyaemia was evident, with abscesses in the myocardium and kidney; in another (30D) hypertrophic polypoid gastritis and an active chronic peptic ulcer of the lower oesophagus were present.

In the abdominal aorta single patchy atheromatous lesions were found in 2 monkeys - fatty deposits in one (30D) and fibrous plaques in the other (12C). No other lesions were present in the main branches of the aorta, coronary, and cerebral arteries.

Ocular pathology. Gross examination of the eye globes showed no noteworthy abnormalities. The microscopic findings of interest were seen at the anterior and peripheral portion of the optic nerve of 2 monkeys $(2 \mathrm{C}, 43 \mathrm{C})$, one of which $(2 \mathrm{C})$ developed pallor of the optic discs during the study. Serial sections from both animals taken through the horizontal plane of the pupil-optic disc axis (Fig. 2) and cross-sections of the optic nerve (Fig. 3) showed segmental lesions where the neuroglial pattern of the optic nerve was disrupted. There was an almost total absence of axons, which was associated with diminution of myelin and the presence of enlarged reactive astrocytes and microglial proliferation. In some of these segmental atrophic lesions cavernous spaces which stained negative for acid mucopolysaccharides were presented (Fig. 4). The central retinal artery and vein were both patent and showed no abnormalities in all sections examined. Frozen sections of the content of the retinal and choroidal vessels of the posterior pole of the eye showed positive staining (oil-red $\mathrm{O}$ stain), indicating a very high lipid level of the plasma. No other vascular or neural changes were observed in the retina.

\section{Discussion}

The method used in our study to produce hyperlipoproteinaemia in rhesus monkeys has previously been shown to be practical and effective, ${ }^{3}$ and the high levels of serum lipid obtained were similar to others reported. ${ }^{1-3}$ However, the severe and prolonged hyperlipoproteinaemia produced in our experiment, unlike other reported studies, ${ }^{34}$ was not associated with any consistent atherosclerotic lesions 


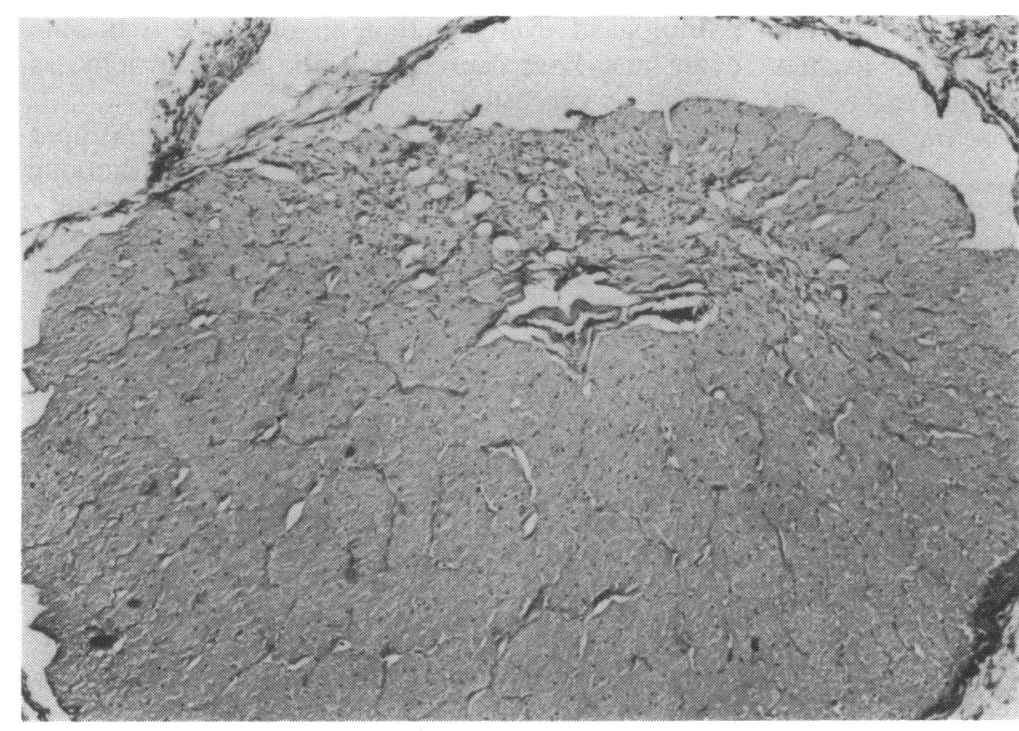

Fig. 3 Segmented atrophy with glial proliferation and cavernous spaces of the optic nerve of a monkey fed high fat-cholesterol diet for 14 months. (Haematoxylin and eosin, $\times 33)$.

of the cerebral vasculature, carotid arteries, thoracic or abdominal aorta and its branches, or coronary arteries, nor were the retinal vessels affected. Thus the normal appearance of the retinal vessels on ophthalmoscopic examination was consistent with the absence of obvious systemic atherosclerosis.

Lipaemia retinalis of varying severity observed throughout the study was related mainly to the plasma triglycerides level, ${ }^{8}$ since triglycerides are chiefly transported in the serum in the form of particles with diameter greater than $1 / 4$ the wave length of visible light (or $0 \cdot 1 \mu \mathrm{m})$. Such large particles, by interrupting the light rays, can be seen in the visible light range and give the serum the appearance of turbidity or lipaemia. ${ }^{9}$ The elevated plasma triglycerides levels of the 6 monkeys studied as well as the lipaemia retinalis depicted by fundal follow-up examinations (Fig. 1) support this concept. Increased cholesterol per se does not render the plasma turbid, ${ }^{10}$ and therefore could not be the cause of lipaemia retinalis. In clinical conditions lipaemia retinalis might be the presenting symptom that indicates the existence of certain types of hyperlipoproteinaemia (type I, III, IV, or V), but it is not found in type II of this disorder. ${ }^{11}$ The earliest

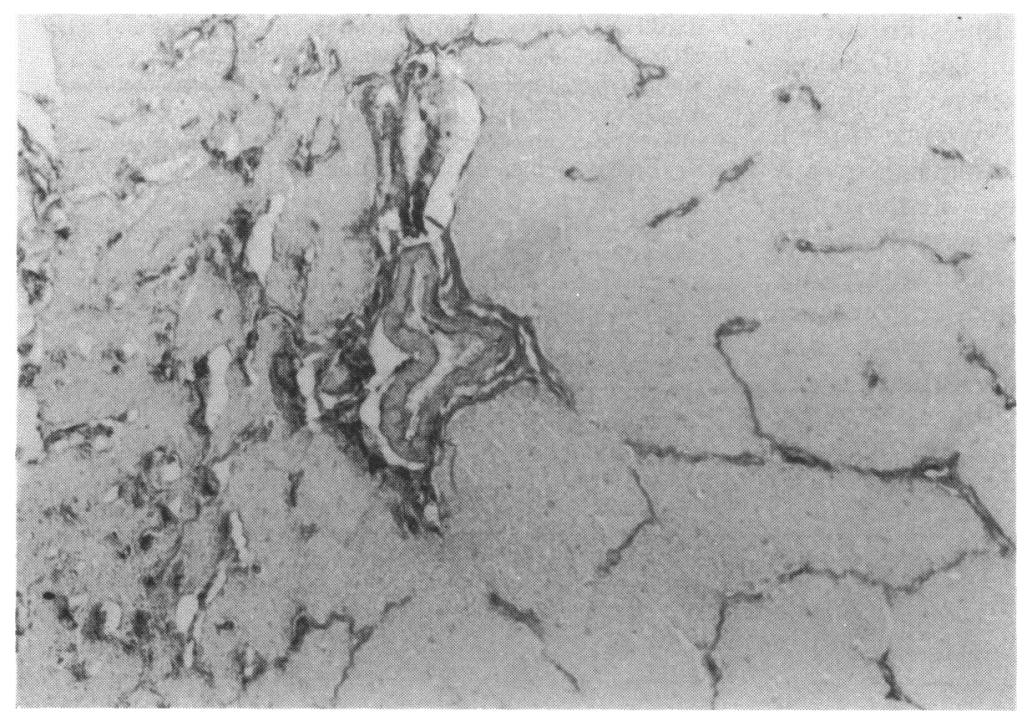

Fig. 4 Field of the optic nerve from the same tissue as Fig. 3 showing cavernous spaces within the segmental atrophic lesions which stained negative for acid mucopolysaccharides. (Luxol fast blue and alcian blue, $\times 150)$. 
signs of lipaemia retinalis are seen in the small peripheral retinal vessels where the centrally located thin column of red blood cells contributes to a better view of the surrounding creamy appearance of the plasma.

Asteroid bodies, as observed in the vitreous of one of the monkeys, are a common finding in clinical diabetes mellitus ${ }^{12} 13$ and have also been reported in primary hypercholesterolaemia. ${ }^{1314}$ Although there is no direct evidence that these asteroids were associated with the severe hyperlipoproteinaemia in our animals, it is unlikely that this feature is mere coincidence.

The most striking pathological findings in this study are the bilateral segmental atrophy and gliosis of the optic nerves in 2 of the 4 monkeys examined at necropsy (Figs. 2, 3). These lesions strongly resemble those found in cases of chronic ischaemic optic neuropathy. These optic nerve lesions, which to the best of our knowledge are reported for the first time in connection with experimental hyperlipoproteinaemia, were associated with embolisation of foam cells in the vessels of the brain in one case and in the penicillated splenic artery in the other case. Analogous localised optic nerve atrophy with similar pathological characteristics, bearing a close resemblance to human ischaemic optic neuropathy, has been reported after experiments with occlusion of the posterior ciliary artery in monkeys. ${ }^{15}$ Other workers, ${ }^{16}$ using the same techniques, reported similar pathological changes of a lesser degree and extent. More recently a clinical report ${ }^{17}$ has indicated that embolic occlusion of pial or pial-derived arterioles may similarly produce segmental infarction of the optic nerve.

Although attribution of the exact aetiology of the anterior ischaemic optic neuropathy seen in our experiment is rendered difficult by the lack of obvious occlusive components in blood vessels nourishing the optic nerve, the coexistence of systemic emboli elsewhere in the body in these animals strongly implicates foam cell embolisation of the small branches of the posterior ciliary arteries or of arteries of the pia mater as an ischaemic pathogenetic mechanism of the optic nerve damage. The presence of lacunae which on staining proved negative for acid mucopolysaccharides, and which were associated with increased glial reaction (Fig. 4), is suggestive histo- pathological evidence that an occlusive ischaemic event may have caused the optic nerve atrophy, as previously suggested. ${ }^{18}$

Our findings imply that a highly abnormal lipid metabolism may cause systemic or ocular embolic effects. Since the studies reported here involved very high levels of serum lipids, comparisons with analogous clinical conditions should be made only with caution.

\section{References}

1 Cox, GC, Taylor CB, Cox LG, Counts MA. Atherosclerosis in rhesus monkeys. Hypercholesterolemia induced by dietary fat and cholesterol. Arch Pathol 1958; 66: 32-52.

2 Taylor CB, Manalo-Estrella P, Cox GE. Atherosclerosis in rhesus monkeys. V. Marked diet-induced hypercholesterolemia with xanthomatosis and severe atherosclerosis. Arch Pathol 1963; 76: 239-49.

3 Younger RK, Scott HW Jr, Butts WH, Stephenson SE Jr. Rapid production of experimental hypercholesterolemia and atherosclerosis in the rhesus monkey: comparation of five dietary regimens. J Surg Res 1969; 9: 263-71.

$4 \mathrm{Kramsch}$ DM, Hollander W. Occlusive atherosclerotic disease of the coronary arteries in monkey (Macaca irus) induced by diet. Exp Mol Pathol 1968; 9: 1-22.

5 Cogan DG, Kuwabara T. Ocular changes in experimental hypercholesterolemia. Arch Ophthalmol 1959; 61: 219-25.

6 Janes RG. Changes in the rabbit's eye caused by cholesterol feeding. Am J Ophthalmol 1964; 58: 819-28.

7 Slatter DH, Nelson AW, Stringer JM. Effects of experimental hyperlipoproteinemia on the canine eye. Exp Eye Res 1979; 29: 437-47.

8 Dunphy EB. Ocular conditions associated with idiopathic hyperlipemia. Am J Ophthalmol 1950; 33: 1579-86.

9 Ahrends EH Jr, Kunkel HG. The stabilization of serum lipid emulsions by serum phospholipids. J Exp Med 1949; 90: 409-24.

10 Albrink MJ, Man EB, Peters JP. The relation of neutral fat to lactescence of serum. J Clin Invest 1955; 34: 147-57.

11 Vinger PF, Sachs BA. Ocular manifestations of hyperlipoproteinemia. Am J Ophthalmol 1970; 70: 563-73.

12 Rutherford CW. Asteroid bodies in the vitreous. Arch Ophthalmol 1933; 9: 106-17.

13 Bard LA. Asteroid hyalitis. Relationship to diabetes and hypercholesterolemia. Am J Ophthalmol 1964; 58: 239-42.

14 Smith JL. Asteroid hyalitis: incidence of diabetes mellitis and hypercholesterolemia. JAMA 1958; 168: 891-3.

15 Hayreh SS, Baines JAB. Occlusion of the posterior ciliary artery. III. Effects on the optic nerve head. Br J Ophthalmol 1972; 56: $754-64$.

16 Anderson DR, Davis EM. Retina and optic nerve after posterior ciliary artery occlusion. An experimental study in squirrel monkeys. Arch Ophthalmol 1974; 92: 422-6.

17 Lieberman MF, Shahi A. Embolic ischemic optic neuropathy. Am J Ophthalmol 1978; 86: 206-10.

18 Zimmerman LE, de Venecia G, Hamasaki DI. Pathology of the optic nerve in experimental acute glaucoma. Invest Ophthalmol Visual Sci 1967; 6: 109-25. 